\title{
EFFECT OF LOW-FREQUENCY NOISE ON ADIABATIC PASSAGE IN A SUPERCONDUCTING NANOCIRCUIT
}

\author{
A. LA COGNATA*, P. CALDARA, D. VALENTI ${ }^{\dagger}$ and B. SPAGNOLO \\ Dipartimento di Fisica e Tecnologie Relative, \\ Università di Palermo, Group of Interdisciplinary, \\ Physics and CNISM-INFM, Unità di Palermo, \\ Viale delle Scienze, Ed.18, \\ I-90128 Palermo, Italy \\ *angelolc@gip.dft.unipa.it \\ †valentid@gip.dft.unipa.it \\ A. D'ARRIGO, E. PALADINO and G. FALCI \\ Dipartimento di Metodologie Fisiche e Chimiche (DMFCI), \\ Università di Catania Viale A. Doria 6, \\ I-95125 Catania, Italy \\ CNR-IMM MATIS, Catania, Italy \\ ${ }^{\ddagger}$ gfalci@dmfci.unict.it
}

Received 24 August 2010

\begin{abstract}
Recent experiments have demonstrated coherent phenomena in three-level systems based on superconducting nanocircuits. This opens the possibility to detect Stimulated Raman Adiabatic Passage (STIRAP) in artificial atoms. Low-fequency noise (often $1 / f$ ) is one of the main sources of decoherence in these systems, and we study its effect on the transfer efficiency. We propose a way to analyze low frequency fluctuations in terms of fictitious correlated fluctuations of external parameters. We discuss a specific implementation, namely the Quantronium setup of a Cooper-pair box, showing that optimizing the trade-off between efficient coupling and protection against noise may allow us to observe coherent population transfer in this nanodevice.
\end{abstract}

Keywords: STIRAP; quantronium; coherent transfer population; Zener transition; three-level system.

PACS Number(s): 74.25.Jb, 73.23.-b, 73.40.Gk, 42.50.Md, 42.65.Dr

\section{Introduction}

The observation of coherent dynamics in nanodevices is an important achievement towards quantum control in solid state devices. In the last decade superconducting nanocircuits exhibiting the dynamics of single 'artificial atoms', ${ }^{1-3}$ two coupled artificial atoms ${ }^{4,5}$ and artificial atoms coupled to electromagnetic resonators ${ }^{6,7}$ have 
been demonstrated. This development opens new perspectives to study quantum phenomena in solid-state devices that traditionally have been part of quantum optics. $^{8}$

So far most of the research in this field has focused on the two lowest level of artificial atoms. In the last few years, it has been proposed that multilevel quantum coherent effects, ${ }^{8-10}$ could be observed in superconducting nanodevices: various schemes have been proposed to observe electromagnetically induced transparency, ${ }^{11}$ and selective population transfer by adiabatic passage. ${ }^{12-16}$ Very recently, few experiments have demonstrated features of multilevel coherence in such devices, as the Autler-Townes effect, ${ }^{17,18}$ coherent population trapping, ${ }^{19}$ electromagnetically induced transparency, ${ }^{20}$ preparation and measurement of three-state superpositions. ${ }^{21}$

In studying quantum optical effects in solid state devices, several differences are encountered with respect to the atomic realm: coupling between subsystems is larger, but also noise is larger, and often extends over several decades, low-frequency noise being the most important source of decoherence in many of the solid state implementations of quantum bits. ${ }^{22,23}$ On the other hand solid state devices offer several design solutions, and the possibility of tuning by external controls the spectral properties of the artificial atom. ${ }^{24}$ All these elements come into play in multilevel structures, ${ }^{25}$ together with new features, as for example selection rules. Differences between specific designs may become crucial for the successful implementation of specific protocols.

In this work we will study coherent population transfer using the STIRAP protocol three-level artificial atoms. In Sec. 2 we introduce STIRAP, and discuss the sensitivity of the transfer efficiency to external parameters. Then we consider a specific implementation of three-level artificial atom based on the Quantronium $\operatorname{design}^{2,26}$ and introduce a model for low-frequency charge noise (Sec. 3). In Sec. 4, we propose a way to characterize the effects of low-frequency noise, reducing the problem to that of the sensitivity of the transfer efficiency to fictitious correlated external parameters.

\section{Coherent Population Transfer in Three-Level Atoms}

In quantum optics, the STIRAP technique is based on a $\Lambda$ configuration (Fig. 1) of two hyperfine ground states $|0\rangle$ and $|1\rangle$ and an excited state $|2\rangle$, with energies $E_{0}, E_{1}$ and $E_{2}$ respectively. ${ }^{8}$ The system is driven by two classical laser fields, ${ }^{9,8}$ called the Stokes laser $\Omega_{12}=\Omega_{s} \cos \omega_{s} t$ and the Pump laser $\Omega_{02}=\Omega_{p} \cos \omega_{p} t$. Each laser is nearly resonant with the corresponding transition. In the usual situations, we can treat the laser drive fields in the Rotating-Wave Approximation (RWA). Moreover, one can introduce a phase transformation of the atomic basis and express the Hamiltonian in a doubly rotating frame, with angular frequencies given by $\omega_{i}$ of the driving fields. The effective Hamiltonian reads

$$
\tilde{H}=\delta|1\rangle\left\langle 1\left|+\delta_{p}\right| 2\right\rangle\langle 2|+\frac{1}{2}\left(\Omega_{s}|2\rangle\left\langle 1\left|+\Omega_{p}\right| 2\right\rangle\langle 0|+\text { h.c. }\right)
$$




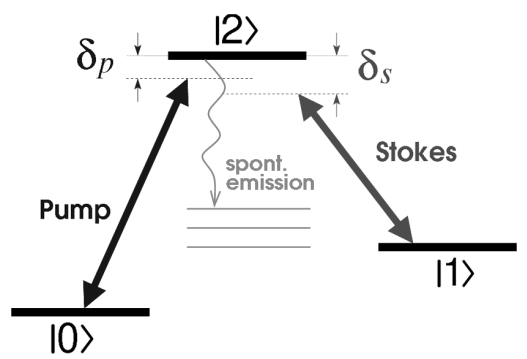

Fig. 1. A three-level atom driven by two lasers in the $\Lambda$ scheme. The state $|2\rangle$ may have a large decay probability.

where we define the detunings $\delta_{s}=E_{2}-E_{1}-\omega_{s}, \delta_{p}=E_{2}-E_{0}-\omega_{p}$ and the twophoton detuning $\delta=\delta_{p}-\delta_{s}=E_{2}-E_{1}-\left(\omega_{p}-\omega_{s}\right)$.

At two-photon resonance, $\delta=0$, the Hamiltonian (1) has an eigenstate which is a superposition of the two lowest atomic levels only

$$
|D\rangle=\frac{1}{\sqrt{\left|\Omega_{s}\right|^{2}+\left|\Omega_{p}\right|^{2}}}\left(\Omega_{s}|0\rangle-\Omega_{p}|1\rangle\right) .
$$

It is usually referred to as the dark state since, despite the presence of the lasers, the atom cannot be excited to the state $|2\rangle$ and consequently decay by spontaneous emission (Fig. 1). Instead, the laser fields interfere destructively and, as a result, the population is coherently trapped. A given dark state can be prepared by an appropriate choice of both the Rabi frequencies $\Omega_{i}$ and the relative phase of the ac fields.

\subsection{The STIRAP protocol}

From Eq. (2), it can be seen that by slowly varying the coupling strengths, $\Omega_{s}(t)$ and $\Omega_{p}(t)$, the dark state can be rotated in the two-dimensional subspace spanned by $|1\rangle$ and $|0\rangle$. Using adiabatic dynamics in the rotating frame, the STIRAP protocol implements coherent population transfer between the atomic states $|0\rangle \rightarrow|1\rangle .^{9}$

The system can be prepared in the state $|0\rangle$ by letting $\Omega_{p}=0$ and switchig on $\Omega_{s}(t) \neq 0$. By slowly switching $\Omega_{s}$ off while $\Omega_{p}(t)$ is switched on, the population can be transferred from state $|0\rangle$ to state $|1\rangle$. Finally also $\Omega_{p}$ is switched off. The mixing angle of the dark state Eq. (2) is defined as $\theta(t)=2 \arctan \left[\Omega_{p}(t) / \Omega_{s}(t)\right]$, and evolves from $\theta=0$ to $\theta=2 \pi$ (Fig. 2, upper right panel).

This is the so-called counterintuitive scheme as opposed to the intuitive strategy where the pump pulse preceeds the Stokes pulse. In this case population transfer involves, as an intermediate step, population of the excited state $|2\rangle$, which can undergo spontaneous decay, strongly affecting the population transfer efficiency. One advantage of STIRAP is that, in the ideal procedure, the state $|2\rangle$ is never populated, $, 9,10$ therefore it is not sensitive to spontaneuos decay. Moreover, provided adiabaticity is preserved, STIRAP is in principle insensitive to many details of the 

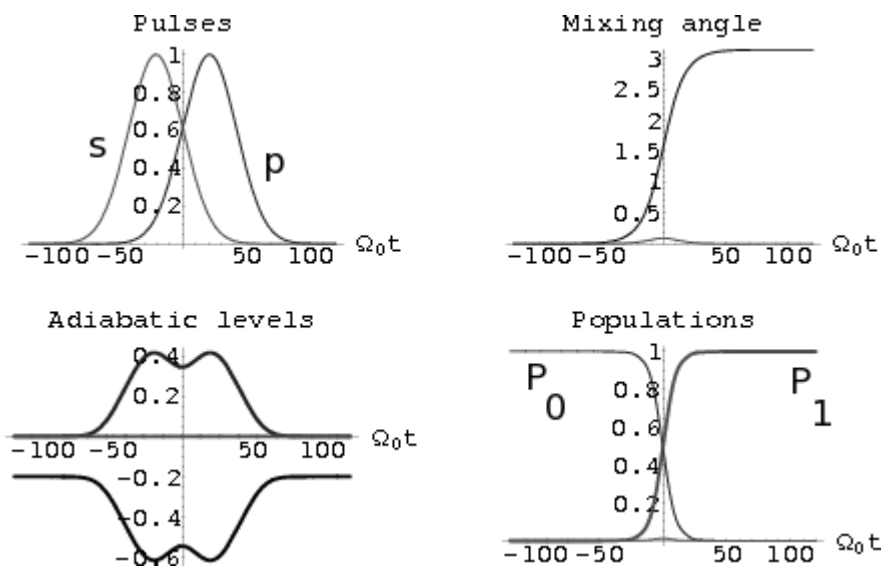

Fig. 2. Ideal STIRAP at two-photon resonance $\delta=0$, obtained by operating with two pulses in the counterintuitive sequence (top left panel). The system prepared in the state $|0\rangle$ follows the Hamiltonian along the zero-energy adiabatic level (left lower panel) yielding complete population tranfer (right lower panel, where $P_{i}=|\langle i \mid \psi(t)\rangle|^{2}$ ). In top right panel, the mixing angle of the dark state as a function of time for the adiabatic evolution. The pump laser is slightly detuned, $\delta_{p}=-0.2 \Omega_{0}$.

protocol, and in practice it turns out to be insensitive to the precise timing of the operations.

\subsection{Sensitivity to parameters}

Adiabaticity is critical to achieve high efficiency, therefore much effort has been devoted in the past to optimization of the pulse shapes. ${ }^{10}$ A necessary condition for adiabaticity is $\left|\Omega_{j} / \Omega_{j}\right| \ll \omega_{j}(j=s, p)$, which suggests that efficiency can be improved by using large enough Rabi peak frequencies. Formally, they determine a large (Autler-Townes) splitting of the instantaneous eigenstates in the rotating frame. ${ }^{10,9}$ This splitting prevents unwanted transitions triggered by off diagonal parts (neglected in the adiabatic approximation) of the Hamiltonian in the instantaneous eigenbasis. These non-adiabatic terms are proportional to $\dot{\theta}(t)$ and tend to detrap the population, reducing the transfer efficiency. If we let $\Omega_{p}(t)=\Omega_{0} f[(t-\tau) / T]$ and $\Omega_{s}(t)=\kappa \Omega_{0} f[(t+\tau) / T]$, a positive delay $\tau$ implements the counterintuitive sequence of STIRAP. For Gaussian pulses, $f(x)=\mathrm{e}^{-x^{2}}$, optimal choices are $\Omega_{0} T>10$ and $\tau \approx T .{ }^{10}$ In this paper we use a reduced pulse width $\Omega_{0} T=30$ and a delay $\tau=0.7 T$.

\subsubsection{Sensitivity to detunings}

When the two frequencies $\omega_{s}$ and $\omega_{p}$ are not exactly resonant with the respective transitions, the presence of non-zero detunings $\delta_{s}$ and $\delta_{p}$ may strongly affect the efficiency. Actually, the two-photon detuning is the crucial parameter. As it is shown in Fig. 3, small deviations of the two photon detuning $\delta$ lead to a substantial decrease of the efficiency, which is less sensitive to single-photon detunings at two-photon resonance $\delta=0$. Actually, phenomena entering non-ideal STIRAP are qualitatively 

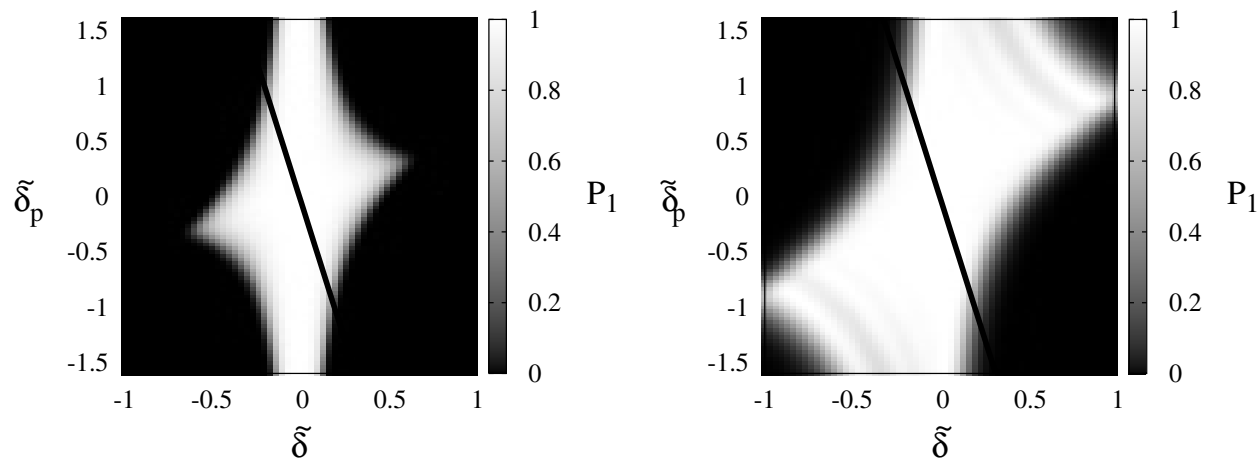

Fig. 3. (left panel) Contour plot of the intensity of the transfer efficiency as a function of single-photon and two-photon detuning for equal peak Rabi frequencies $\kappa=\Omega_{S} / \Omega_{P}=1$ (left panel) and $k=2$ (right panel). In axes $\mathrm{x}, \mathrm{y}$ we have $\tilde{\delta}=\delta / \Omega_{0}$ and $\tilde{\delta}_{p}=\delta_{p} / \Omega_{0}$, respectively. In both panels, the bright region corresponds to large efficiency of population transfer (more than $80 \%$. A two-photon detuning $|\delta| \gtrsim \Omega_{0} / 5$ determines a substantial decrease of the efficiency. The line corresponds to correlated detunings, which give an effective description of fluctuation in the Quantronium (Sec. 4). Increasing the strength of the Stokes pulses enlarges asymmetrically the region of large transfer efficiency.

different according to $\delta$ vanishing or not, and their interplay leads to a rich physical picture.

Finite single photon detunings at $\delta=0$ do not affect the formation of the dark state, because the mixing angle does not depend on it. Instead they increase the nonadiabatic terms. ${ }^{10}$ The efficiency is insensitive to small single-photon detunings $\left(\delta \lesssim \Omega_{0}\right.$, see also Fig. 2), while larger ones prevent the adiabatic follow on of the dark state.

The detuning from two-photon resonance is more detrimental for STIRAP, because it prevents the exclusive population of the trapped state, which is no longer an instantaneous eigenstate of the Hamiltonian. A more detailed analysis of the instantaneous eigenstates when $\delta \neq 0$ shows that there is no adiabatic transfer state providing an adiabatic connection from the initial to the target state, as does the dark state for $\delta=0$. In this case, the evolution leads to complete population return of the system to its initial state. The only mechanism which leads to population transfer is by non-adiabatic transitions between the adiabatic states. Actually for small values of $\delta$, narrow avoided crossings between the instantaneous eigenvalues can occur and the population can be transferred by Landau-Zener tunneling, ${ }^{10,9}$ as shown in Fig. 4 .

The above considerations lead to the conclusion that the correlations between the detunings $\delta_{s}$ and $\delta_{p}$ are very important. In fact, strongly correlated fluctuating detunings, nearly preserving two-photon resonance, still allow large transfer efficiency. ${ }^{27}$ This issue becomes very important in the discussion of the effects of lowfrequency noise in solid state nanodevices.

\subsubsection{Sensitivity to Rabi frequencies}

For ideal STIRAP, it is better to have two nearly equal peak Rabi frequencies, i.e. $\kappa=\Omega_{S} / \Omega_{P}=1$. Indeed if the two maximum Rabi frequencies are different, say 

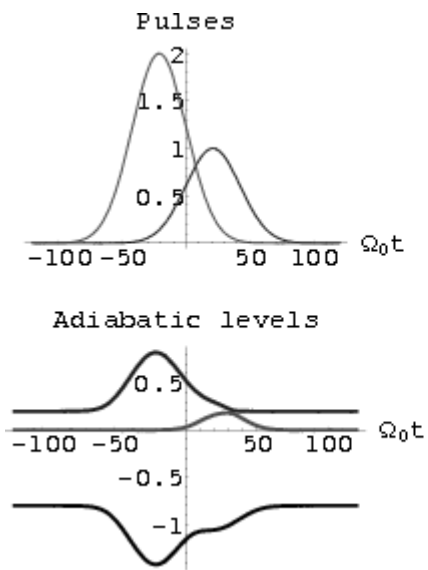
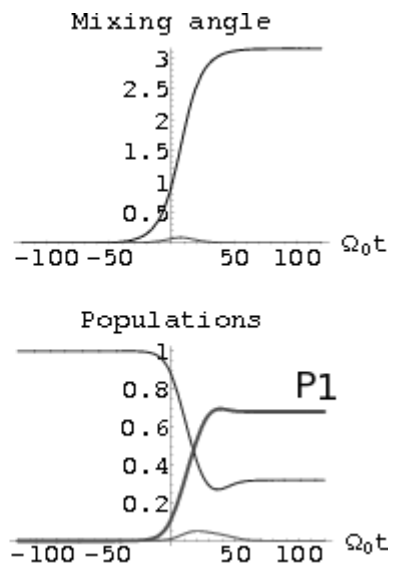

Fig. 4. STIRAP with finite two-photon detuning $\delta=0.2 \Omega_{0}$, with the two pulses in sequence in top left panel. Population transfer occurs due to Zener transitions between crossing adiabatic levels (lower left panel), and the transfer efficiency is reduced (lower right panel). In top right panel, the mixing angle as a function of time. Here $\kappa=2$ and $\delta_{p}=-4 \delta$. This parametrization being appropriate for discussing effects of low-frequency noise in the Quantronium (Sec. 4).

$\kappa>1$, while the pulse widths are about the same, the projection of the state vector onto the adiabatic transfer state is very good initially (because in our case the more intense pulse occurs first), but necessarily less good in the final stage. Consequently, the transfer efficiency will be small. ${ }^{10}$

The situation may be different if finite detuning is considered. In particular in the right panel of Fig. 3 it is shown that the region of great transfer efficiency enlarges asymmetrically. This happens when the larger pulse occurs during the Zener process of imperfect STIRAP (the opposite situation is illustrated in Fig. 4).

Of course, using large pulse areas, small deviations from the optimal conditions do not lead to significant drop in transfer efficiency, and in general increasing both the amplitudes is the convenient strategy to counteract the effect of imperfections. However, in solid state nanodevices there are restrictions on the amplitude and symmetry of the coupling to the microwave fields, playing the role of the lasers. Therefore, operating at $\kappa \neq 1$ may give room to further optimize the transfer efficiency.

\section{STIRAP in the Quantronium}

We now discuss the implementation of the Hamiltonian (1) in the Quantronium. ${ }^{2}$ The basic unit of this device consists of a Cooper pair box, namely a superconducting loop interrupted by two adjacent tunnel junctions with Josephson energies $E_{J} / 2$ (Fig. 5). The two small junctions define the superconducting island of the box, whose total capacitance is $C$ and charging energy $E_{C}=(2 e)^{2} / 2 C$. The electrostatic energy can be modulated by a gate voltage $V_{g}$ connected to the island via a capacitance 


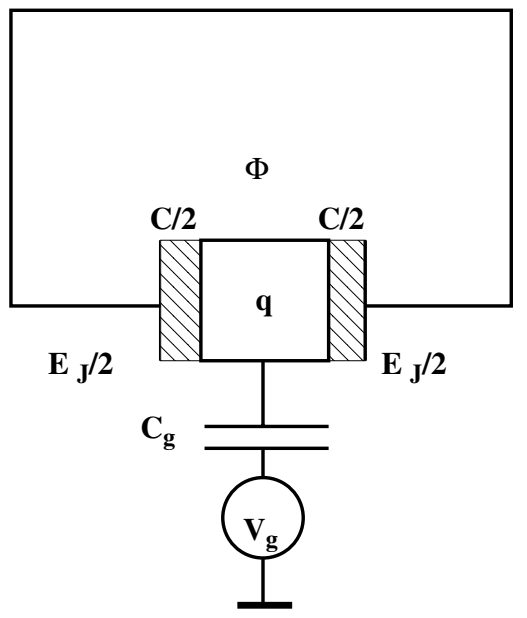

Fig. 5. Equivalent circuit for the Quantronium. Here $q$ and $C$ are the charge and the capacitance of the superconducting island respectively; $C_{g}$ and $V_{g}$ are the capacitance and the voltage of the gate; $E_{g}$ is the Josephson energy and $\Phi$ is the magnetic flux.

$C_{g} \ll C$ and the Hamiltonian reads

$$
H_{0}\left(q_{g}\right)=\sum_{q} E_{C}\left[q-q_{g}\right]^{2}|q\rangle\langle q|-\frac{E_{J}}{2}(|q\rangle\langle q+1|+\text { h.c. }),
$$

where $\{|q\rangle\}$ are eigenstates of the number operator $\hat{q}$ of extra Cooper pairs in the island. We have defined the reduced gate charge $q_{g}=C_{g} V_{g} /(2 e)$, which is the control parameter of the system. Eigenstates of the box are superpositions of charge eigenstates. The spectrum can be modified by tuning $q_{g}$ (Fig.6) and the device is usually operated as a qubit close to the value $q_{g}=1 / 2$. This is a symmetry point for the
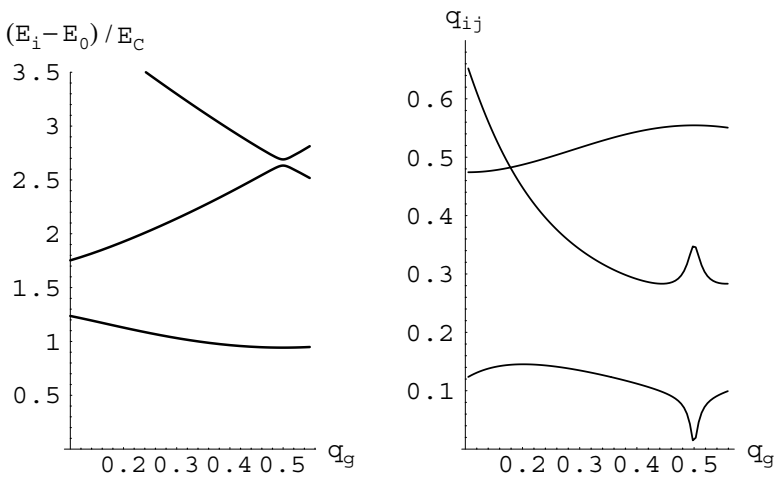

Fig. 6. Left panel: energy spectrum of a Quantronium setup with $E_{J}=E_{C}$. The splitting $E_{i}-E_{0}$ in units of $E_{C}$ is plotted as functions of $q_{g}$, The first splitting is given by $E_{1}(1 / 2)=0.94$. Right panel: off-diagonal entries of the Cooper pair number operator, $q_{01}, q_{12}$ and $q_{02}$ from top to bottom. 
device Hamiltonian (3) and it turns out that it is an optimal working point where the system is well protected against external noise, allowing to obtain experimental dephasing times of several hundreds nanoseconds. ${ }^{2,26}$

Manipulation of the quantum state is performed by adding to the dc part of the gate voltage, ac microwave pulses with small amplitudes $q_{g} \rightarrow q_{g}+q_{g}^{a c}(t)$. The resulting Hamiltonian can be written as

$$
H_{\text {tot }}(t)=H_{0}\left(q_{g}\right)+A(t) \hat{q},
$$

where $A(t)=-2 E_{C} q_{g}^{a c}(t)$. The effective three-level artificial atom Hamiltonian

$$
H(t)=\sum_{i} E_{i}\left|\phi_{i}\right\rangle\left\langle\phi_{i}\left|+A(t) \sum_{i j} q_{i j}\right| \phi_{i}\right\rangle\left\langle\phi_{j}\right|
$$

is obtained by projecting $H_{\text {tot }}(t)$ onto the subspace spanned by the three lowest energy eigenvectors $\left|\phi_{i}\right\rangle, i=0,1,2$ of $H_{0}\left(q_{g}\right)$. In Eq. (5) $q_{i j}=\left\langle\phi_{i}|\hat{q}| \phi_{j}\right\rangle$. The STIRAP protocol can be carried out if we let $A(t)=A_{s}(t) \cos \omega_{s} t+A_{p}(t) \cos \omega_{p} t$. We then use the RWA, by retaining only quasi-resonant off-diagonal and co-rotating terms of the drive Hamiltonian, which simplifies to

$$
\begin{aligned}
A(t) \hat{q} \rightarrow H_{R W A}(t)= & \frac{1}{2} q_{12} A_{s}(t) \mathrm{e}^{i \omega_{s} t}\left|\phi_{1}\right\rangle\left\langle\phi_{2}\right| \\
& +\frac{1}{2} q_{02} A_{p}(t) \mathrm{e}^{i \omega_{p} t}\left|\phi_{0}\right\rangle\left\langle\phi_{2}\right|+\text { h.c. }
\end{aligned}
$$

In this approximation the truncated Hamiltonian (5) is transformed to the doubly rotated frame, at angular frequencies $\omega_{s}$ and $\omega_{p}$. This yields an effective Hamiltonian $\tilde{H}\left(q_{g}\right)$ with the structure of Eq. (1), which therefore implements the $\Lambda$ configuration. Notice that matrix elements $q_{i j}=\left\langle\phi_{i}|\hat{q}| \phi_{j}\right\rangle$ play the same role of the dipole matrix elements in defining the Rabi frequencies, $\Omega_{s}=q_{12} A_{s}$ and $\Omega_{p}=q_{02} A_{p}$.

The RWA of Eq. (6) is justified in the regime where peak Rabi frequencies are much smaller than the splittings, $\Omega_{i} \ll\left|E_{i}-E_{j}\right|$, which is the usual experimental regime. In this case the terms neglected are rapidly oscillating in the rotating frame, and only produce a small and fast modulation in the dynamics. The approximation is supported by simulations of the full Hamiltonian (4), using more than ten energy levels ${ }^{15,28}$ for the usual operating region near $q_{g}=1 / 2$.

It is worth stressing the dependence of the effective Hamiltonian $\tilde{H}\left(q_{g}\right)$ on the bias charge $q_{g}$. For instance in Eq. (1) detunings depend on $q_{g}$ via the energies $E_{i}$ and peak Rabi frequencies via off diagonal matrix elements $q_{i j}$ (see Fig. 6). In particular at the symmetry point, $q_{g}=1 / 2$, the matrix element $q_{02}$ vanishes and in general selection rules hold preventing transitions between energy states with the same parity of the label. The off-diagonal matrix elements $q_{i j}$ shown in Fig. 6 play the same role of the dipole matrix elements in atoms. The largest one is $q_{01}$, which provides the coupling for qubit operations. Fields in STIRAP are coupled via $q_{12}$ and $q_{02}$. This latter vanishes due to a parity selection rule at the symmetry point $q_{g}=1 / 2$. 


\subsection{Broadband noise}

Since the nanocircuit is not isolated, the model has to be supplemented with noise terms. The structure of coupling to noise can be understood considering classical fluctuations of each of the parameters in the Hamiltonian of Eq. (3). For instance fluctuations of the gate charge can be accounted for by adding a classical stochastic term $q_{g} \rightarrow q_{g}+\delta q_{x}(t)$. Physical processes described by these fluctuations are those leading to a stray electrical polarization of the island, and include effects of voltage fluctuations of the circuit and effects of switching impurities ${ }^{22}$ located in the oxides and in the substrate close to the device. Since these latter are in practice the main source of decoherence (circuit fluctuations can be reduced by careful filtering) for the Quantronium, we will only consider fluctuations of the gate charge, thus acting on the same port used to drive the system. We may write the resulting Hamiltonian as

$$
H=H_{0}\left(q_{g}\right)+H_{R W A}(t)+\delta H
$$

where $\delta H=-2 E_{C} \delta q_{x}(t) \hat{q}$. In general, noise is due to the coupling of the device to an environment which is itself a quantum system, and the Hamiltonian is obtained by letting $\delta H=\hat{X} \hat{q}+H_{\text {env }}$, where $H_{\text {env }}$ describes the environment and $\hat{X}$ is an environment operator. This model allows to treat high-frequency noise by a quantum optical master equation in the weak coupling regime. However the power spectrum of noise in the solid state has a large low-frequency component which invalidates the weak coupling approach. A multistage approach has been proposed ${ }^{23}$ where high and low-frequency noise are separtated, and the latter is treated as an adiabatic classical field. Formally $\hat{X} \rightarrow \hat{X}_{f}-2 E_{C} \delta q_{x}(t)$, where $\hat{X}_{f}$ describes fast environmental degrees of freedom and $\delta q_{x}(t)$ is now a classical slow stochastic process. We let $q_{x}(t)=$ $q_{g}+\delta q_{x}(t)$ and write the Hamitonian as

$$
H=H_{0}\left(q_{x}(t)\right)+H_{R W A}(t)+\hat{X} \hat{q}+H_{\mathrm{env}} .
$$

In many cases low-frequency noise with $1 / f$ spectrum, which is the leading contribution of the slow dynamics of $q_{x}(t)$, is captured by a Static-Path Approximation (SPA), that is approximating the stochastic process by a suitably distributed random variable. ${ }^{23,26}$ In the case of many weakly coupled noise sources, the distribution of $\delta q_{x}$ is characterized by an energy width $\sigma=2 E_{C} \sigma_{x}$. Populations and coherences are obtained by averaging over this distribution the entries of the reduced density matrix of the system. This approach has quantitatively explained the power law decoherence observed in Quantronium ${ }^{26}$ and in phase qubits, ${ }^{32}$ and has been recently studied for optimal tuning of multiqubit systems. ${ }^{25}$

This point of view provides a simple argument explaining why the symmetry point $q_{g}=1 / 2$ is well protected against external noise. Indeed, since the energy splitting $E_{1}-E_{0}$ depends only quadratically on the fluctuations $\delta q_{x}$ around this point, energy fluctuations are suppressed. As a consequence, superpositions of the two lowest energy levels keep coherent, yielding a power law suppression of the $\operatorname{signal}^{23,26}$ and longer dephasing time. 


\subsection{Effective model for low-frequency noise in STIRAP}

In order to study STIRAP we project the Hamiltonian (7) on the subspace spanned by the three lowest energy instantaneous eigenvectors of $H_{0}\left(q_{x}(t)\right)$. In doing so we assume the adiabaticity of the dynamics induced by $\delta q_{x}(t)$, which allows to neglect effects of the time-dependence of the eigenvectors. Of course, if we start from the SPA version of the Hamiltonian (7), this condition is automatically verified. We focus on the system plus drive Hamiltonian, $H_{0}\left(q_{x}(t)\right)+H_{R W A}(t)$, which has in the rotated frame the same structure of Eq. (1). Parameters entering the Hamiltonian depend, of course, on the realization of the stochastic process. Fluctuations of the eigenenergies translate in fluctuations of the detunings (we let $E_{0}=0$ )

$$
\delta\left(q_{x}\right)=E_{1}\left(q_{x}\right)-\omega_{p}+\omega_{s} ; \quad \delta_{p}\left(q_{x}\right)=E_{2}\left(q_{x}\right)-\omega_{p} .
$$

Also the effective drive fluctuates due to fluctuations of the charge matrix elements, for instance $\Omega_{p}=q_{02}\left(q_{x}\right) A_{p}$.

In the regime of validity of the SPA, this analysis shows that the effect of lowfrequency noise in solid-state devices, can be discussed in term of sensitivity of the transfer efficiency obtained by STIRAP to parameters characterizing an equivalent drive. This allows to apply several results known from quantum optics to solid state devices. For instance the large sensitivity to two-photon detuning, translates in the sensitivity to fluctuations of the lowest splitting, which is then the main figure to be minimized in order to achieve efficient population transfer in the solid state. Notice also that, the main steps of the analysis carried out for the Quantronium can also be applied to other solid state implementations devices, as long as decoherence in the dynamics of the two lowest energy levels is well-characterized.

\section{Effects of Low-Frequency Noise in the Quantronium}

In this section we will apply the above analysis to discuss the observability of STIRAP in the Quantronium, and we will consider a device with $E_{J}=E_{C}$, whose spectral properties are given in Fig. 6. An important point is that while dephasing is minimized by operating at the symmetry point $q_{g}=1 / 2$, the selection rule $q_{02}=0$ prevents to implement STIRAP. Therefore, it has been proposed to operate slightly off the symmetry point.

In these conditions it has been shown that STIRAP allows a substantial coherent population transfer also in the presence of high-frequency noise. Notice that, while in quantum optical systems STIRAP connects two ground states, in solid state devices high-frequency noise leads to decay $1 \rightarrow 0$. These processes are well characterized experimentally. ${ }^{26}$ In Ref. 15 it has been shown that secular dephasing between the above two states does not produce relevant effects during population transfer. A careful analysis ${ }^{28}$ has allowed us to optimize parameters for STIRAP in the presence of high-frequency noise, showing that operating at $q_{g}=0.47$ already provides sufficient coupling $q_{02}$. 
On the other hand, it is known that the effect of low-frequency noise increases when the system is operated away from the symmetry point. ${ }^{26,33}$ This opens the question of the trade-off between efficient coupling of the driving fields and dephasing due to slow excitations in the solid-state. In this work we focus on this issue and we neglect high-frequency noise.

Another consequence of the selection rule is that, in the vicinity of the symmetry point, coupling with the drives is asymmetric. At $q_{g} \approx 0.47$ we have $q_{02} \approx q_{12} / 4$ (see Fig. 6). Since in any case it is convenient to work with the largest pump pulse Rabi peak frequency $\Omega_{0}$, we will fix this value. It can be estimated by writing $\Omega_{0}=$ $\left(q_{02} / q_{01}\right) \Omega_{R} \approx \Omega_{R} / 6$, where $\Omega_{R}$ is the maximal angular frequency for Rabi oscillations between the lowest doublet. Figures of approximately $\nu_{R}=750-900 \mathrm{MHz}$ can be achieved in the Quantronium, corresponding to a maximum field amplitude $A_{p}$ yielding $\nu_{p}=100-150 \mathrm{MHz}$. The peak Rabi frequency of the Stokes field could be chosen as $\nu_{s}=\kappa \nu_{p}$, with $\kappa \leq 4$, but we will argue that $\kappa=1$ is the optimal choice.

Fluctuations $\delta q_{x}$ of the gate charge can be estimated from the dephasing time of the qubit at the symmetry point. This is due to energy fluctuations $\sigma / E_{1}(1 / 2) \sim 0.01$. Therefore fluctuations of gate charge, which are characteristic of the environment only, are estimated by $\sigma_{x}=\sigma /\left(2 E_{C}\right) \approx 3 \cdot 10^{-3}$, where we used $E_{C} \sim 15 \mathrm{GHz}$. Notice that these features may depend on details of the protocol as the total measurement time, but for $1 / f$ noise the dependence is logarithmic and improving the procedure does not bring essential changes of $\sigma_{x}$.

We choose to operate at single and two-photon resonance, $\delta=\delta_{p}=0$ at $q_{g}=0.47$. According to Eq. (8), fluctuations $\delta q_{x}$ determine a distribution of the detuning. In the left panel of Fig. 6 , we can directly read off fluctuations of the splitting, which give the estimate $\Delta \delta=\Delta E_{1}\left(q_{x}\right) \approx\left(\partial E_{1} / \partial q_{x}\right)_{q_{g}} \delta q_{x}$ and $\Delta \delta_{p}=\Delta E_{2}\left(q_{x}\right) \approx\left(\partial E_{2} / \partial q_{x}\right)_{q_{g}} \delta q_{x}$.

Therefore, fluctuations of the detunings are anticorrelated, $\Delta \delta_{p}=a \Delta \delta$, where the ratio of the two derivatives is given by $a \approx-5$. This corresponds to the lines drawn in the efficiency diagrams of Fig. 3. Using $\left(\partial E_{1} / \partial q_{x}\right)_{q_{q}} \delta q_{x} \approx\left(E_{J} / 4\right)$, we find that fluctuations of the two-photon detuning are estimated by $\sigma_{\delta} / \Omega_{0} \approx E_{J} \sigma_{x} /\left(4 \Omega_{0}\right)$ $\approx \sigma /\left(8 \Omega_{0}\right) \sim 0.1-0.2$, identifying the region of the efficiency diagrams explored by the system during the protocol. This estimate suggests that energy fluctuations in the Quantronium should still allow to observe coherent population.

Fluctuations of the off-diagonal elements can be estimated by the plots in Fig. 6 (right panel), yielding figures of $\sim(1 / 4) \sigma_{x} \Omega_{0} \sim 10^{-3} \Omega_{0}$, therefore they can be neglected. The transfer efficiency is then calculated by averaging the population histories over the distribution of correlated detunings. Results are shown in Fig. 7 for different values of the fluctuation intensity of the two-photon detuning $\sigma_{\delta}$ in units of $\Omega_{0}$. Here detunings are anticorrelated $\left(\delta_{p}=-5 \delta\right)$ and drives have been symmetrized $(\kappa=1)$, by using a lower amplitude $A_{s}$ for the Stokes field. It is seen that in standard experimental conditions the low-frequency noise allows from $60 \%$ to more than $90 \%$ population transfer in the Quantronium. Notice that even for $\sigma_{\delta}=0.2 \Omega_{0}$ the average population of the intermediate level is very small during the whole procedure. 


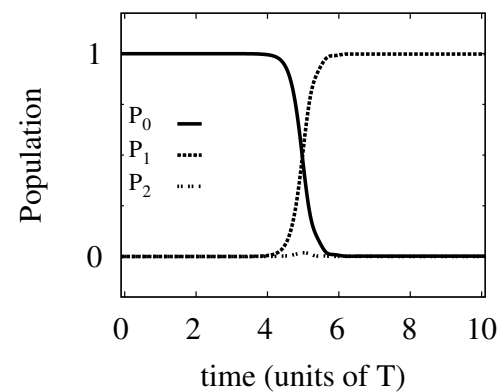

(a)

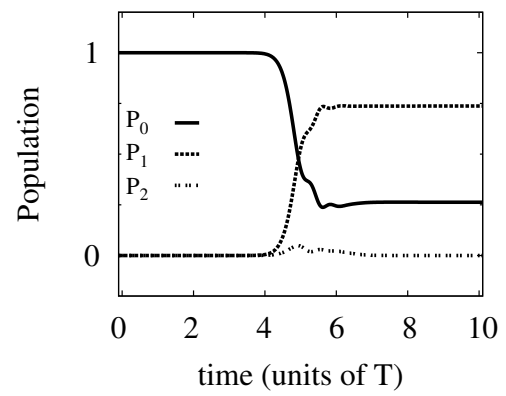

(c)

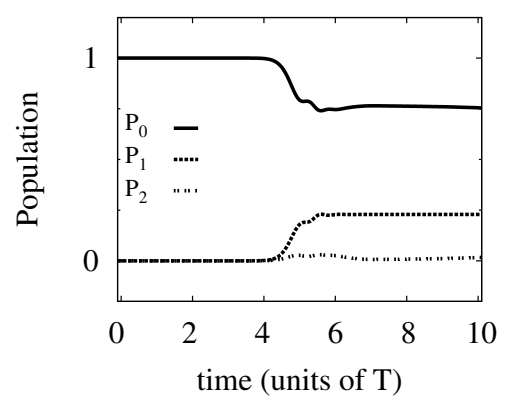

(e)

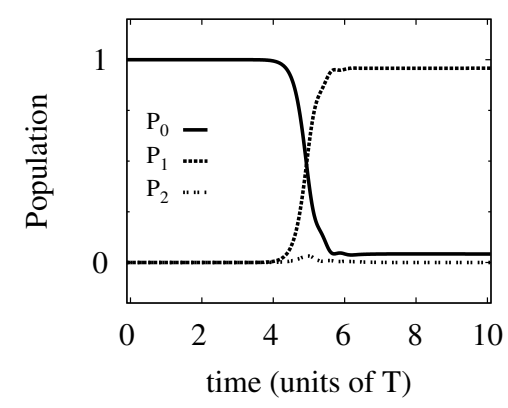

(b)

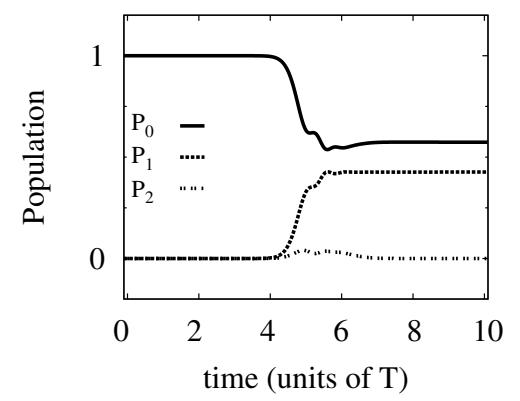

(d)

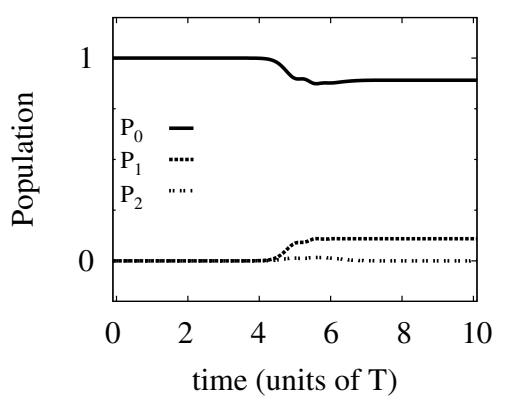

(f)

Fig. 7. Averaged population histories for different values of the fluctuation intensity of the two-photon detuning, $\sigma_{\delta}$. In panels (a)-(f), we have $\sigma_{\delta}=0.05,0.1,0.2,0.4,0.8,1.6$ in units of $\Omega_{0}$, respectively. Here detunings are anticorrelated $\left(\delta_{p}=-5 \delta\right)$ and drives have been symmetrized $(\kappa=1)$ by using a lower amplitude $A_{s}$ for the Stokes field. For $\Omega_{0}=2 \pi \cdot 10^{8} \mathrm{rad} / \mathrm{s}$ the relevant curve is $\sigma_{\delta}=0.2$ and $T=48 \mathrm{~ns}$ yielding $60 \%$ of population transfer. Slightly increasing $\nu_{p}=150 \mathrm{MHz}$ one obtains $\sigma_{\delta}=0.125$ and $T=30$ ns.

Finally we comment about the optimization of the laser amplitudes. In the above simulations we used $\kappa=1$, but it would be possible to use a larger Stokes pulse, up to $\kappa=4$. However this does not improve the efficiency if fluctuations of the detunings are anticorrelated. As shown in Fig. 8, in this case the region of large efficiency shrinks for increasing $\kappa$. 


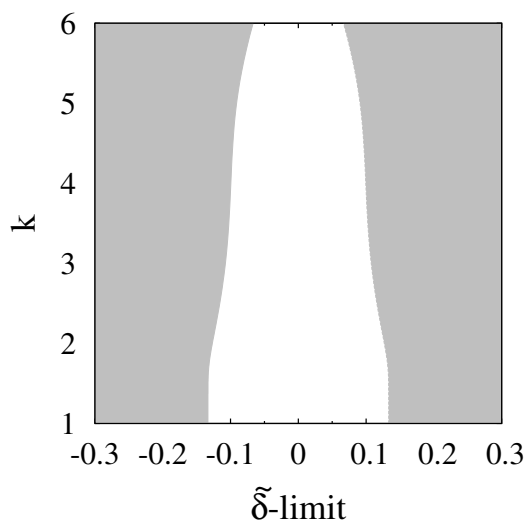

Fig. 8. Ratio of the maximum drive amplitudes $k=\Omega_{S} / \Omega_{P}$ as a function of the two-photon detuning limits, $\tilde{\delta}=\delta / \Omega_{0}$, for anticorrelated noise, typical of Quantronium $\left(\delta_{p}=-5 \delta\right)$. The white zone is the region where we have more than $80 \%$ of transfer efficiency of STIRAP.

\section{Conclusion}

We studied the effect of low-frequency noise on the transfer efficiency of STIRAP, proposing that low-frequency fluctuations of the spectrum can be analyzed in terms of fictitious correlated fluctuations of the detunings. For solid-state noise with large low-frequency component (e.g. for $1 / f$ noise) the leading effect (SPA approximation) is equivalent to consider statistically distributed detunings and can be discussed by analyzing the sensitivity to parameters of the protocol.

We applied the theory to the Quantronium, showing that correlated fluctuations of the energy splittings have to be considered, and that transfer efficiency is mainly sensitive to decoherence in the subspace of the two-lowest levels, which is wellcharacterized experimentally. Selection rules prevent to work at the symmetry point, where decoherence is minimal. Therefore, the observation of coherent population transfer requires optimization of the trade-off between increasing coupling and greater sensitivity to low-frequency noise. We have shown that this is indeed possible, given the measured figures of low-frequency noise.

Notice that we have used pulses of width $T=48-30 \mathrm{~ns}$. Therefore, the total time of the protocol $\sim 200-350 \mathrm{~ns}$ is longer than the dephasing time of the qubit, as determined solely by static inhomogeneities. This dephasing time is smaller off the symmetry point (in the experiment of Ref. 26 the dephasing time for coherent oscillations dropped from $T_{\phi} \sim 600 \mathrm{~ns}$ at the symmetry point to $T_{\phi} \sim 50 \mathrm{~ns}$ at $\left.q_{g}=0.47\right)$. This shows that STIRAP is less sensitive than coherent oscillations to low-frequency noise. Actually, accounting for high frequency noise the process will be limited by the relaxation $T_{1} \gtrsim 500 \mathrm{~ns}$.

The analysis we illustrated applies as well to other superconducting nanodevices. In particular, it could allow one to design correlations of fluctuations of the energy spectrum, which maximize the Zener channel of population transfer. 


\section{References}

1. Y. Nakamura, Yu. Pashkin and J. S. Tsai, Nature 398 (1999) 786.

2. D. Vion, A. Aassime, A. Cottet, P. Joyez, H. Pothier, C. Urbina, D. Esteve and M. H. Devoret, Science 296 (2002) 886.

3. I. Chiorescu, Y. Nakamura, C. J. P. M. Harmans and J. E. Mooij, Science 299 (2003) 1869.

4. T. Yamamoto, Yu. A. Pashkin, O. Astafiev, Y. Nakamura and J. S. Tsai, Nature 425 (2003) 941.

5. J. B. Majer, F. G. Paauw, A. C. J. ter Haar, C. J. P. M. Harmans and J. E. Mooij, Phys. Rev. Lett. 94 (2005) 090501.

6. A. Wallraff, D. I. Schuster, A. Blais, L. Frunzio, R. S. Huang, J. Majer, S. Kumar, S. M. Girvin and R. J. Schoelkopf, Nature 431 (2004) 162.

7. I. Chiorescu, P. Bertet, K. Semba, Y. Nakamura, C. J. P. M. Harmans and J. E. Mooij, Nature 431 (2004) 159.

8. M. O. Scully and M. S. Zubairy, em Quantum Optics (Cambridge Univ. Press, Cambridge 1997).

9. K. Bergmann, H. Theuer and B. W. Shore, Rev. Mod. Phys. 70 (1998) 1003.

10. N. V. Vitanov, T. Halfmann, B. W. Shore and K. Bergmann, Annu. Rev. Phys. Chem. 52 (2001) 763.

11. K. V. R. M. Murali, Z. Dutton, W. D. Oliver, D. S. Crankshaw and T. Orlando, Phys. Rev. Lett. 93 (2004) 087003.

12. M. H. S. Amin, A. Yu. Smirnov and A. Maassen v.d. Brink, Phys. Rev. B 67 (2003) 100508(R).

13. J. Siewert and T. Brandes, Adv. Solid State Phys. 44 (2004) 181.

14. Y.-X. Liu, J. Q. You, L. F. Wei, C. P. Sun and F. Nori, Phys. Rev. Lett. 95 (2005) 087001.

15. J. Siewert, T. Brandes and G. Falci, Opt. Comm. 264 (2006) 435; J. Siewert, T. Brandes and G. Falci, Phys. Rev. B 79 (2009) 024504, arXiv:cond-mat/0509735v1.

16. M. Mariantoni, M. J. Storcz et al., arXiv:cond-mat/0509737v2 (2005).

17. M. A. Sillanpää, J. Li, K. Cicak, F. Altomare, J. I. Park, R. W. Simmonds, G. S. Paraoanu and P. J. Hakonen, Phys. Rev. Lett. 103 (2009) 193601.

18. M. Baur, S. Filipp, R. Bianchetti, J. M. Fink, M. Göppl, L. Steffen, P. J. Leek, A. Blais and A. Wallraff, Phys. Rev. Lett. 102 (2009) 243602.

19. W. R. Kelly, Z. Dutton, J. Schlafer, B. Mookerji, T. A. Ohki, J. S. Kline and D. P. Pappas, Phys. Rev. Lett. 104 (2010) 163601.

20. A. A. Abdumalikov et al., arXiv 1004.2306v1 quant-ph, Phys. Rev. Lett. (2010).

21. R. Bianchetti, S. Filipp, M. Baur, J. M. Fink, C. Lang, L. Steffen, M. Boissonneault, A. Blais and A. Wallraff, arXiv:1004.5504v1.

22. E. Paladino, L. Faoro, G. Falci and R. Fazio, Phys. Rev. Lett. 88 (2002) 228304.

23. G. Falci, A. D'Arrigo, A. Mastellone, and E. Paladino, Phys. Rev. Lett. 94 (2005) 167002; E. Paladino et al., Phys. Scr. T 137 (2009) 014017.

24. G. Falci and R. Fazio, in G. Casati, D. L. Shepeliansky, P. Zoller, G. Benenti Eds., Quantum Computer, Algorithms and Chaos. pp. 363-416, IOS press, ISBN/ISSN: 1-58603-660-2.

25. E. Paladino et al., Phys. Rev. B 81 (2010) 052502.

26. G. Ithier, E. Collin, P. Joyez, P. J. Meeson, D. Vion et al., Phys. Rev. B 72 (2005) 134519 .

27. L. P. Yatsenko, V. I. Romanenko, B. W. Shore and K. Bergmann, Phys. Rev. A 65 (2002) 043409; P. A. Ivanov, N. V. Vitanov and K. Bergmann, Phys. Rev. A 70 (2004) 063409 .

28. G. Mangano, J. Siewert and G. Falci, Eur. Phys. Journ. ST 160 (2008) 259. 
29. A. S. Parkins, P. Marte, P. Zoller and H. J. Kimble, Phys. Rev. Lett. 71 (1993) 3095.

30. M. Henrich, T. Legero, A. Kuhn and G. Rempe, Phys. Rev. Lett. 85 (2000) 4872.

31. M. A. Kmetic, R. A. Thuraisingham and W. J. Meath, Phys. Rev. A 33 (1986) 1688.

32. S. Poletto et al., New Jour. Phys. 11 (2009) 013009.

33. G. Falci, A. Mastellone, A. D'Arrigo and E. Paladino, Open Sys. É Inf. Dyn. 13 (2006) 323. 